\title{
Early Epidemiological and Survival Characteristics of COVID-19 Patients Undergoing Cardiovascular Surgery
}

\author{
Saeed Sadeghian ${ }^{1}$, seyed khalil forouzannia ${ }^{1}$, jamshid bagheri ${ }^{1}$, Namvar Movahedi ${ }^{1}$, khosro \\ barkhordari $^{1}$, Hassan Soltani nia ${ }^{1}$, kaveh hosseini ${ }^{2}$, Mojgan Ghavami ${ }^{1}$, Masoumeh \\ Lotfi-Tokaldany $^{2}$, Mohammad Ali Boroumand ${ }^{1}$, Mina Pashang ${ }^{1}$, Shahrzad Salehbeigi ${ }^{1}$, and \\ Seyedeh Hamideh Mortazavi ${ }^{1}$ \\ ${ }^{1}$ Tehran University of Medical Sciences \\ ${ }^{2}$ Affiliation not available
}

May 27, 2020

\begin{abstract}
background and aim: world health organization (WHO) declared the novel coronavirus 2019 outbreak a pandemic on 11 March 2020. No data have been published so far regarding the COVID-19 patients undergoing cardiovascular surgery. Methods: Medical files of 503 patients undergoing cardiovascular surgery were evaluated. Sixty-four patients (12.7\%) was diagnosed as COVID-19 cases after the surgery following getting symptomatic or detection of abnormality in their laboratory results. The valve surgery in one positive COVID-19 case was canceled due to his abnormal laboratory finding and suspicion to COVID-19. The patients' clinical course and survival status were documented and analyzed. Results: Coronary artery bypass graft (CABG) was the most type of surgery in both positive and negative COVID-19 groups. In-hospital mortality was demonstrated in 6 $(9.4 \%)$ and $10(2.3 \%)$ patients in positive and negative COVID-19 groups, respectively. CABG had been performed in five COVID-19 deaths while one of the expired cases underwent valve surgery. The median of white blood cell (WBC) count and C-reactive protein (CRP) was higher and the percentage of lymphocyte was lower in patients who expired. Conclusions: We recommend to postpone all elective surgeries till the end of the crisis and limit the surgeries to only emergency situations. In these cases, the patient should be screened and get necessary medications if highly suspected. In borderline surgical indications, especially in individuals with history of contact to infection, we suggest to screen for COVID-19 before the surgery and monitor the patients closely afterward. All health-care staffs should use full personal protective equipment.
\end{abstract}

\section{Background}

Severe acute respiratory syndrome coronavirus 2 (SARS-COV-2) which is nominated as novel coronavirus disease (COVID 2019) by world health organization (WHO), has affected various countries around the world $^{1}$. On 11 March 2020, WHO declared the coronavirus outbreak a pandemic and involved various countries around the world ${ }^{2-5}$. Iran, the second largest country in the Middle East also officially announced the first case of the disease. According to the available studies, different patients with COVID-19 have been evaluated descriptively and in some investigations prognostic factors have been identified ${ }^{6-8}$, however no data have been published regarding the patients with COVID-19 undergoing cardiovascular surgery so far. In this study, we aimed to evaluate clinical course of patients undergoing cardiovascular surgery at the time of the COVID-19 epidemic at a high-volume cardiac tertiary center.

\section{Method}

In this cross sectional study, the medical files of 503 patients undergoing cardiovascular surgery (January 21, 2020 - April 18, 2020) at Tehran Heart Center (THC) ${ }^{9}$, a cardiac tertiary center, affiliated to Tehran University of Medical Sciences, were evaluated, retrospectively. Among these individuals, 64 patients (12.7\%) 
had been diagnosed as COVID-19 cases after the surgery by Reverse transcription polymerase chain reaction (RT-PCR [Nanjing Vazyme Medical Technology Co., Ltd]) and chest computed tomography (CT) scan following getting symptomatic or detection of abnormality in laboratory results. However, the surgery was canceled before induction of the anesthesia in one of these patients who was candidate for valve surgery, because the operation team got suspected to COVID-19 due to his abnormal complete blood count (CBC) findings. Hence this patient was excluded from the study. The patients' demographic information, cardiovascular risk factor, past medical history, laboratory data, surgery type, computed tomography (CT) scan findings, left ventricular ejection fraction (LVEF) and survival status were documented.

The study protocol was approved by local review board and ethical committee of the hospital and all authors adhered to Helsinki's statement.

\section{Statistical analysis}

Categorical variables were expressed as frequency and percentage. Continuous variables were presented as mean with standard deviation (SD) or median with interquartile range (IQR). All statistical analyses were performed using IBM SPSS Statistics for Windows, version 23.0 (Armonk, NY: IBM Corp).

\section{Results}

In the present study, a total of 503 patients had undergone cardiovascular surgery between January 21, 2020 and April 18, 2020. Coronary artery bypass graft (CABG) was the most type of surgery (63.4\%) followed by valve surgery (12.1\%) in these patients. Among whom, 64 individuals $(12.7 \%)$ were found to be positive COVID-19 according to the findings of RT-PCR and chest CT scan. As shown in Figure 1, among positive COVID-19 individuals, 6 patients (9.4\%) expired at the hospital while in the negative COVID-19 group, 10 $(2.3 \%)$ in-hospital mortalities were documented.

Table 1 demonstrates baseline demographic characteristics and survival status of patients undergoing cardiovascular surgery according to COVID-19 findings. As shown, most of the patients in the two groups were male, and CABG was the most type of surgery in these groups. Valve+other surgery $(14.1 \%)$ was the second most type of surgery in positive COVID-19 group while in negative COVID-19 group, valve surgery $(13.5 \%)$ was the second most type of surgery in negative COVID-19 patients. As mentioned before, the rate of in-hospital mortality was higher in positive COVID-19 groups as compared to negative COVID-19 group $(9.4 \%$ versus $2.3 \%)$.

Table 2 presents the demographic, clinical, surgical and laboratory data of patients with positive COVID-19 undergoing cardiovascular surgery based on survival status. Among 64 cardiovascular surgery patients with positive COVID-19 result, 58 patients (90.6\%) survived and 6 individuals (9.4\%) expired. Men accounted the most of the patients in both survived and expired groups. Among expired patients, one underwent valve surgery and the other 5 patients underwent CABG. In positive COVID-19 patients who survived, CABG and valve+other surgeries were the most type of operations. Fever, chest pain and dry cough was the three most symptoms in both groups. Leukocytosis and lymphopenia were both present in the two survived and expired groups. However, the median $\left(25^{\text {th }}-75^{\text {th }}\right.$ IQR) of white blood cell (WBC) count was higher in patients who expired $(13.66 \times 103 / \mu \mathrm{L}[10.90-22.18]$ versus $11.11 \times 103 / \mu \mathrm{L}$ [10.00-14.00]) while the percentage of lymphocyte was lower in these individuals (13.2\% [9.11-15.15] versus $17.0 \%$ [13.66-22.20]). C-reactive protein (CRP) was high in two groups although the findings were higher in the expired group (15.3 mg/L [3.03-29.96] versus 5.6 $\mathrm{mg} / \mathrm{L}$ [3.72-9.00]). All of the patients showed chest CT scan features of compatible with COVID-19.

\section{Discussion:}

To our knowledge, this is the first report of cardiovascular surgery patients who have been identified as positive COVID-19 cases.

We found that patients with SARS-COV-2 who underwent surgery had 4 times higher in-hospital mortality rate as those who did not have COVID-19 (9.4\% versus $2.3 \%$ ). CABG was the most type of surgery in both 
groups of survived and expired positive COVID patients. In all of our patients, as has been mentioned in the literature, lymphopenia and increased CRP was one of the most findings ${ }^{10,11}$.

In our center, on 5 March 2020; two weeks after the official announcement of COVID-19 in the country by ministry of health and medical education; the THC management team decided to implement a special protocol for patients referring to the hospital including suspending all elective procedures. Till the mentioned date, elective surgeries were being performed and all 6 positive COVID-19 mortality cases in this study, had undergone elective surgeries and they were diagnosed as COVID-19 cases after the operation following getting symptomatic or detection of blood test abnormalities.

After 5 March 2020, all elective surgeries were canceled according to the hospital's management instructions and cardiovascular surgeries were limited to only emergency situations. Before implementing this protocol, there were patients who were admitted at the hospital one month before their surgery being done and they were probably infected during or after the operation and then underwent COVID-19 testing following getting symptomatic or development of respiratory distress.

As described before, in one of our patients, the surgery was canceled before induction of the anesthesia, because the operation team got suspected to COVID-19 due to the patient's weird symptoms and his abnormal complete blood count (CBC) findings.

\section{Conclusion:}

According to our observations and dramatic higher in-hospital mortality of patients with COVID-19 who undergo cardiovascular surgery, we recommend to postpone all elective surgeries till the end of the crisis. In case of emergency indication for surgery, the patients should be tested for RT-PCR and chest CT scan before surgery and get necessary medications if highly suspected to COVID-19. In borderline surgical indications, especially in individuals with history of contact with any confirmed or suspected COVID-19 patients, we suggest to perform laboratory and imaging evaluations, wait for the screening results before the surgery and monitor the patients closely after the surgery. Meanwhile all health-care staffs who are involved in any procedures should use full personal protective equipment.

\section{Funding statement:}

None

\section{Declaration of Interests:}

The authors declare no conflict of interests.

Table 1- Baseline demographic characteristics and survival status of patients undergoing cardiovascular surgery (January 21, 2020 - April 18, 2020) according to COVID-19 results

\section{Age}

Gender (male)

Surgery type

CABG

Valve

CABG + Valve

CABG + Other

Valve + Other

CABG + Valve + Other

Other
Table 1- Baseline demographic characteristics and survival status of patients undergoing cardiovascular surgery (January 21, 2020 - April 18, 2020) according to COVID-19 results

COVID + $(\mathrm{n}=64)$

$61.1(13.82)$

$48(75)$

$39(60.9)$

$2(3.1)$

$8(12.5)$

$1(1.6)$

$9(14.1)$

$2(3.1)$

$3(4.7)$
Table 1- Baseline demographic characteristics and survival status of patients undergoing cardiovascular surgery (January 21, 2020 - April 18, 2020) according to COVID-19 results

COVID - $(\mathrm{n}=439)$

$60.5(11.22)$

$308(70.2)$

$280(63.8)$

$59(13.4)$

$33(7.5)$

$15(3.4)$

$33(7.5)$

$5(1.1)$

$14(3.2)$ 
Table 1- Baseline demographic characteristics and survival status of patients undergoing cardiovascular surgery (January 21, 2020 - April 18, 2020) according to COVID-19 results

In-hospital Mortality Data are shown as number (percent) or mean (SD). CABG; Coronary artery bypass graft
Table 1- Baseline demographic characteristics and survival status of patients undergoing cardiovascular surgery (January 21, 2020 - April 18, 2020) according to COVID-19 results

$4(9.4)$
Data are shown as number
(percent) or mean (SD). CABG;
Coronary artery bypass graft

Table 1- Baseline demographic characteristics and survival status of patients undergoing cardiovascular surgery (January 21, 2020 - April 18, 2020) according to COVID-19 results $10(2.3)$ Data are shown as number (percent) or mean (SD). CABG; Coronary artery bypass graft

Table 2- Baseline demographic, clinical, surgical and laboratory information of patients with positive COVID-19 undergoir

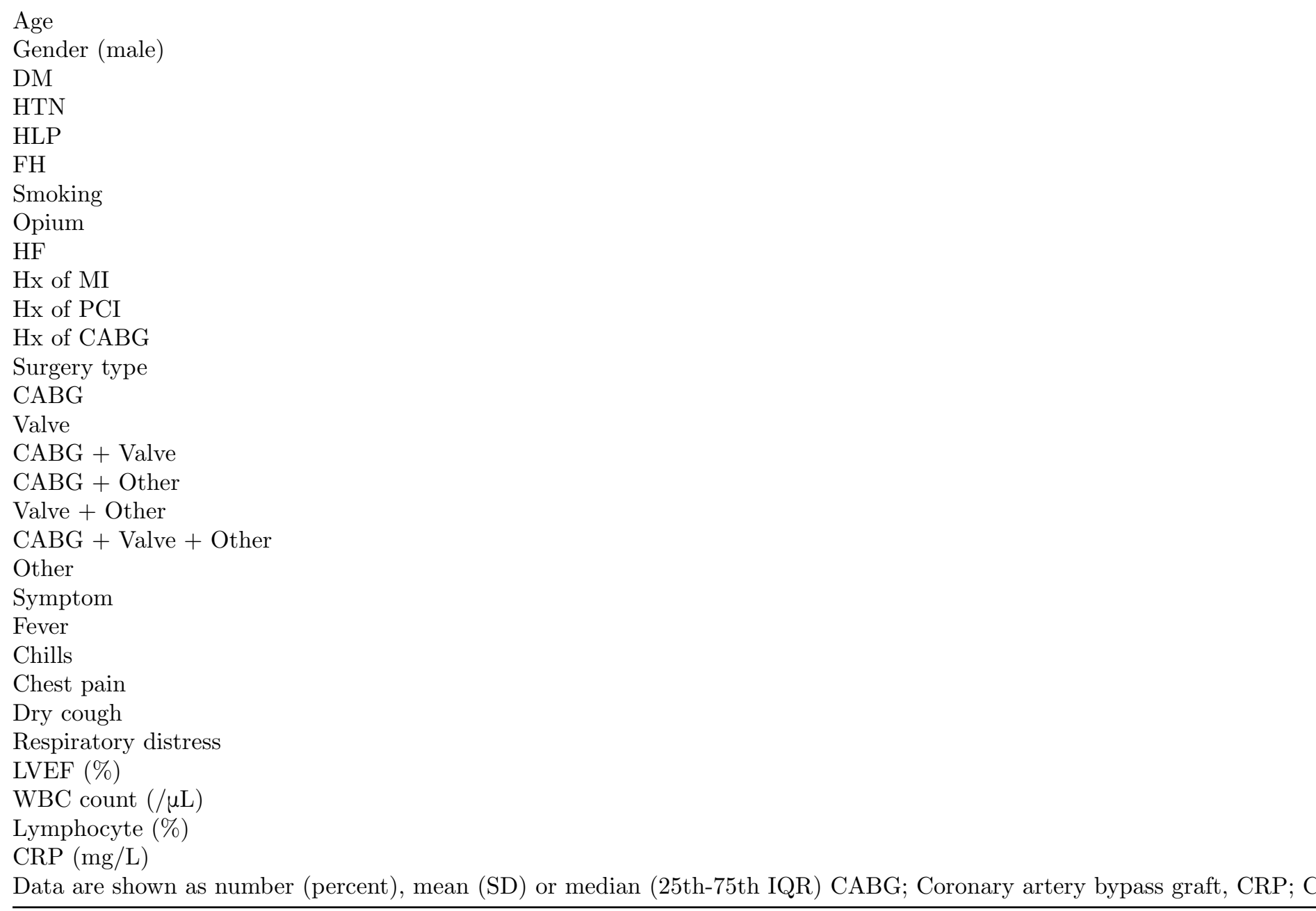




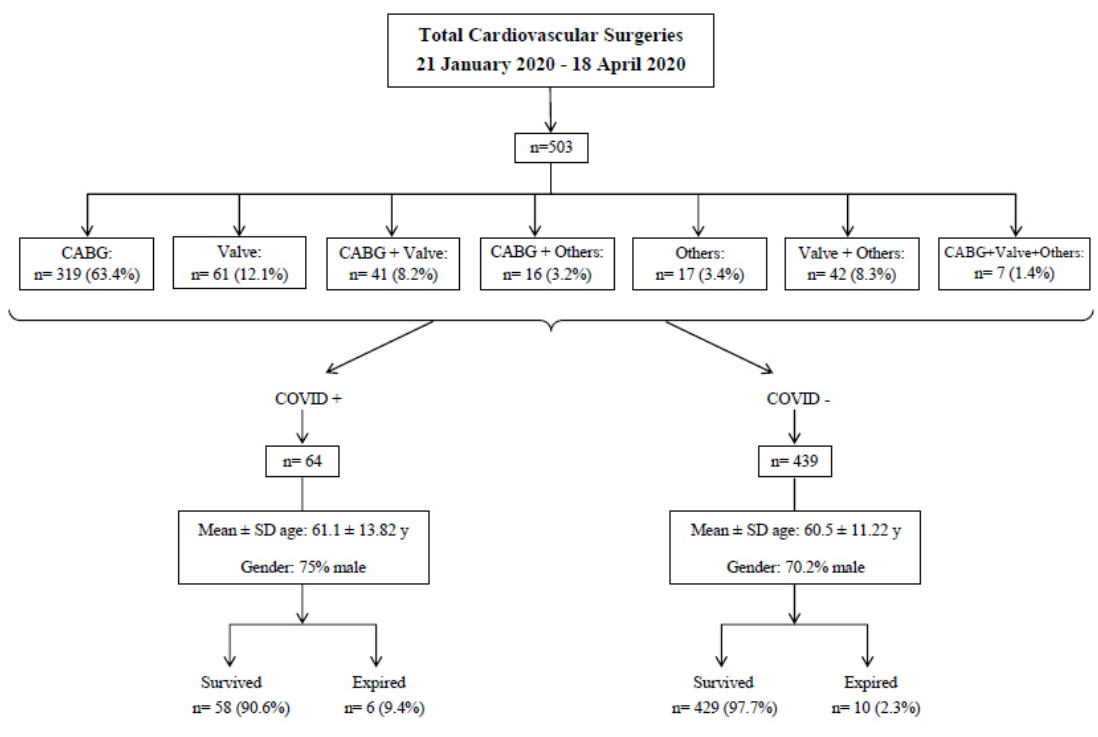

Figure 1- study flow diagram

\section{References:}

1. WHO. Coronavirus disease 2019 (COVID-19) Situation Report - 70. 30 March 2020.

2. Li R, Pei S, Chen B, et al. Substantial undocumented infection facilitates the rapid dissemination of novel coronavirus (SARS-CoV2).Science. 2020.

3. Wu Z, McGoogan JM. Characteristics of and important lessons from the coronavirus disease 2019 (COVID19) outbreak in China: summary of a report of 72314 cases from the Chinese Center for Disease Control and Prevention. Jama. 2020.

4. Zheng Y-Y, Ma Y-T, Zhang J-Y, Xie X. COVID-19 and the cardiovascular system. Nat Rev Cardiol. 2020:1-2.

5. Guo Y, Huang Y, Huang J, et al. COVID-19 pandemic: global epidemiological trends and China's subsequent preparedness and responses. Zhonghua Liu Xing Bing Xue Za Zhi. 2020;41(5):643.

6. Wang L, He W, Yu X, et al. Coronavirus Disease 2019 in elderly patients: characteristics and prognostic factors based on 4-week follow-up. J INFECTION. 2020.

7. Koo JR, Cook AR, Park M, et al. Interventions to mitigate early spread of SARS-CoV-2 in Singapore: a modelling study. Lancet Infect Dis. 2020.

8. Guo T, Fan Y, Chen M, et al. Cardiovascular implications of fatal outcomes of patients with coronavirus disease 2019 (COVID-19).JAMA Cardiol. 2020.

9. Poorhosseini H, Abbasi SH. The Tehran Heart Center. EUR HEART J. 2018;39(29):2695-2696.

10. Tan L, Wang Q, Zhang D, et al. Lymphopenia predicts disease severity of COVID-19: a descriptive and predictive study. SIGNAL TRANSDUCT TAR. 2020;5(1):1-3.

11. Huang C, Wang Y, Li X, et al. Clinical features of patients infected with 2019 novel coronavirus in Wuhan, China. Lancet.2020;395(10223):497-506.

\section{Figure Legends:}

Figure 1- study flow diagram 


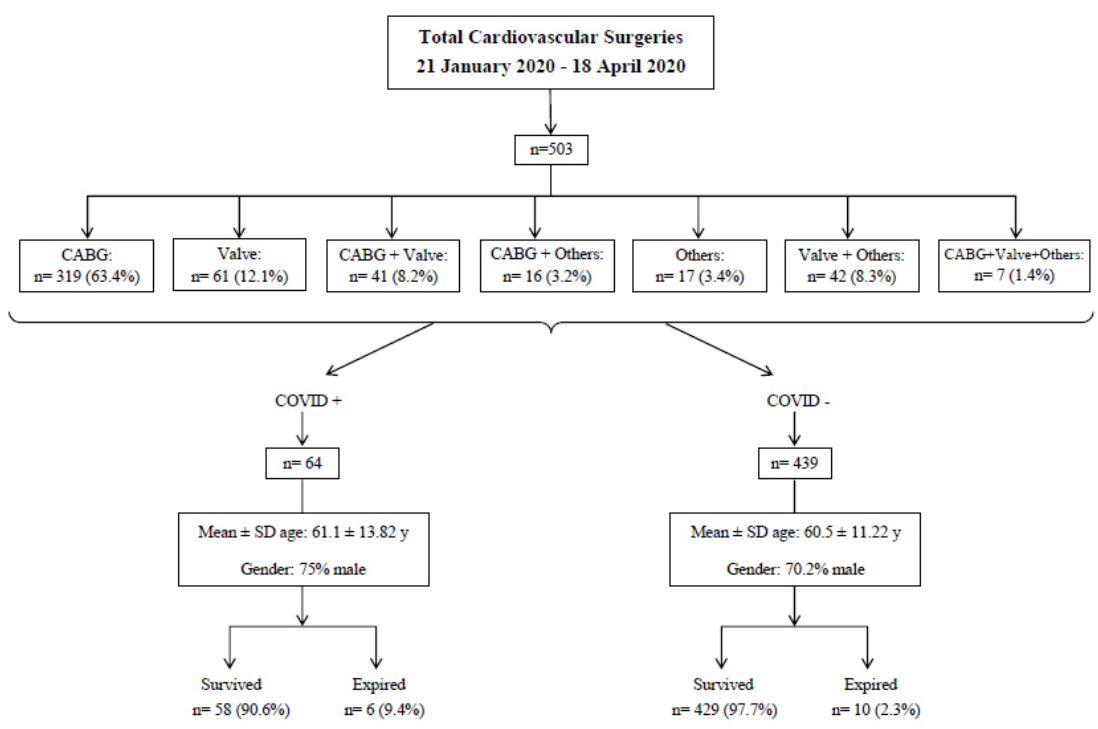

